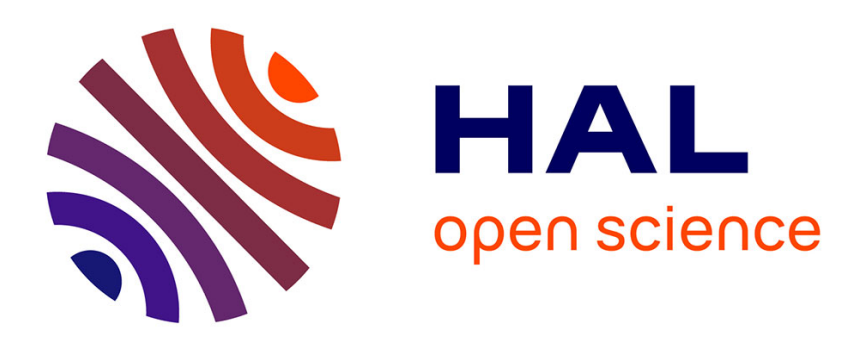

\title{
Des éducateurs à contre courant entre mémoire et histoire
}

\author{
Patricia Bessaoud-Alonso
}

\section{To cite this version:}

Patricia Bessaoud-Alonso. Des éducateurs à contre courant entre mémoire et histoire. Les Études sociales, 2010, 152, pp.171 - 183. 10.3917/etsoc.152.0171 . hal-01385360

\section{HAL Id: hal-01385360 \\ https://hal-unilim.archives-ouvertes.fr/hal-01385360}

Submitted on 5 Oct 2021

HAL is a multi-disciplinary open access archive for the deposit and dissemination of scientific research documents, whether they are published or not. The documents may come from teaching and research institutions in France or abroad, or from public or private research centers.
L'archive ouverte pluridisciplinaire HAL, est destinée au dépôt et à la diffusion de documents scientifiques de niveau recherche, publiés ou non, émanant des établissements d'enseignement et de recherche français ou étrangers, des laboratoires publics ou privés. 


\section{DES ÉDUCATEURS À CONTRE COURANT ENTRE MÉMOIRE ET HISTOIRE}

Patricia Bessaoud-Alonso

Société d'économie et de science sociales | «Les Études Sociales »

2010/2 n 152 | pages 171 à 183

ISSN 0014-2204

Article disponible en ligne à l'adresse :

http://www.cairn.info/revue-les-etudes-sociales-2010-2-page-171.htm

\section{Pour citer cet article :}

Patricia Bessaoud-Alonso, « Des éducateurs à contre courant entre mémoire et histoire », Les Études Sociales 2010/2 (n 152), p. 171-183.

DOI 10.3917/etsoc.152.0171

Distribution électronique Cairn.info pour Société d'économie et de science sociales.

(C) Société d'économie et de science sociales. Tous droits réservés pour tous pays.

La reproduction ou représentation de cet article, notamment par photocopie, n'est autorisée que dans les limites des conditions générales d'utilisation du site ou, le cas échéant, des conditions générales de la licence souscrite par votre établissement. Toute autre reproduction ou représentation, en tout ou partie, sous quelque forme et de quelque manière que ce soit, est interdite sauf accord préalable et écrit de l'éditeur, en dehors des cas prévus par la législation en vigueur en France. Il est précisé que son stockage dans une base de données est également interdit. 


\title{
Des éducateurs à contre courant Entre mémoire et histoire
}

\author{
Patricia BESSAOUD-ALONSO*
}

Lors de la journée d'études du 16 octobre 2009 «L'éducation spécialisée en Algérie et au Maroc avant et après les indépendances 1950-1965 " organisée par le CNAHES et le CIRCEFT ${ }^{1}$ Paris 8, j’ai animé d'une manière très souple, c'est à dire en laissant la parole des intervenants circuler sans trop de dirigisme de ma part, le plateau témoin "Des éducateurs venus de la métropole dans les années 1950 : cadres, experts, militants, pour quelles missions? " composé de Paule Gauneau, Jacques Ladsous et André Heinrich'2

Mon travail de recherche sur les enjeux éducatifs des mémoires algériennes coloniales et post-coloniales ${ }^{3}$ se confrontait, entre autres, à la problématique que posent la notion de mémoire et son rapport à l'histoire, à travers l'élaboration du récit mémoriel et historique chez les sujets.

C'est dans l'avant et dans l'après-coup que je situe l'écriture de ce texte. L'avant pour interroger ce qui constitue la mise en récit de la mémoire des acteurs et l'ancrage historique par lequel il a été traversé et fondé. L'après-coup comme une construction de l'événement dans ce qu'il produit socialement et subjectivement dans sa narration pour les individus. Comment construisent-ils puis restituent-ils leur propre réalité mémorielle à partir d'une histoire sociale-

* Maître de conférences en sciences de l'éducation, Dynadiv-FRED, Université de Limoges.

1. Conservatoire national des archives et de l'histoire de l'éducation spécialisée et de l'action sociale et Centre interdiciplinaire de recherche " culture, éducation, formation, travail *.

2. Respectivement assistante sociale et éducateurs.

3. Patricia BESSAOUD-ALONSO, Les enjeux éducatiff des mémoires algeriennes coloniales et postcoloniales: fabrication et construction des subjectivités, Thèse pour le doctorat en sciences de l'éducation, université Paris 8, 2008. Voir aussi, du même auteur: "Mémoire familiale, mémoire collective de l'Algérie coloniale. Une histoire du silence ", Claude FILTEAU, Michel Benamiano (dir.), Mémoire et culture, Limoges, PULIM, 2006, pp. 497-511; « Figure du père, figure du silence, non-dit familial et étatique à propos de la décolonisation algérienne ", Revue Internationale de la clinique lacanienne, Parentalités d'aujourd'hui et d'ailleurs, $\mathrm{n}^{\circ} 12$, Erès, 2007, pp. 95-99. 
ment produite et partagée et au regard de ce que l'État met en place dans des contextes socio-historiques précis.

J'ai d'abord approché les témoins à travers la lecture de documents écrits relatant une histoire. Pas n'importe laquelle, celle du temps passé en Algérie et au Maroc à l'époque de leur jeunesse en tant que professionnels, fraîchement formés, de l'éducation spécialiste et du travail social. Puis, j'ai écouté le récit oral, à partir de quelques questions, sous forme d'entretiens téléphoniques. De ces deux moments distincts dans leur temporalité et leur spatialité a émergé une rencontre, celle de la mémoire et de l'histoire. Pour les témoins, dans la transmission de ce passé énoncé dans leur présent et moi même dans le recueil, la restitution et l'analyse à élaborer pour signifier ce que je qualifie d'interpénétration des mémoires et de l'histoire.

La première remarque a été la résonance de leurs discours comme une musique familière et prégnante du passé et d'un présent encore assez proche, celui de l'Algérie coloniale, abandonnée depuis le bouclage de ma thèse. Abandon éphémère prêt à resurgir à l'évocation de la ville d'Alger. Cependant, la connaissance, la proximité de ce territoire ne s'est avérée que d'une utilité partielle pour appréhender des trajectoires professionnelles qui me sont assez étranges.

L'étrangeté émane des individus eux mêmes. Ceux que je ne connais pas. Ceux qui ont, un jour, fait le voyage à contre-sens d'une rive à l'autre à la veille des turpitudes de l'histoire.

\section{De la mémoire à l'histoire}

La notion de mémoire est complexe, polysémique et soumise à bien des controverses épistémologiques. Toutefois, la mémoire est un champ d'investigation pour le chercheur dans ce qu'il met en œuvre dans sa quête d'élucidation du passé. Un passé qui s'inscrit dans le présent des individus et participe à leur construction subjective et sociale. La mémoire archaïque ${ }^{4}$ se fonde sur la négociation et la réappropriation par l'individu de son histoire familiale, personnelle et collective produite dans un temps donné de l'histoire. Ce temps de l'histoire n'est pas anodin. Il fabrique des souvenirs identifiables et porteurs d'un contexte précis, en partie à l'insu de celui qui les intériorise, comme une forme de confusion entre ce qui est l'histoire et la construction d'une mémoire socialement partagée. De cette confusion, ou plus justement de ce mélange savant, émerge une parole, un récit qui fait sens pour celui qui l'énonce. De cette énonciation ne naît pas une confrontation entre un discours profane et un discours savant. C'est à dire un discours de vérité, un discours de la preuve d'une part et un

4. Anne MUXEL, Individu et mémoire familiale, Paris, Nathan, 1996. 
discours à ajuster et soumis aux affects, aux implications libidinales d'autre part. L'intérêt, me semble t-il, est dans l'interstice. Il est dans les effets que produisent les récits. Dans le temps de l'histoire différentes étapes se chevauchent et se croisent. L'histoire immédiate n'a pas d'existence, elle a un vécu. C'est seulement l'événement traumatique, violent, singulier, qui donne conscience de sa réalité. Cette réalité prend forme dans la construction de deux récits : celui de l'histoire et celui de la mémoire. Le récit de l'histoire s'élabore dans la mise à distance de l'objet à décrypter, à mettre au jour et dans l'étude des traces que les hommes qui font l'événement nous ont laissées. La mémoire convoque un récit partiel soumis à la subjectivation ${ }^{5}$ et socialement construit. Cette subjectivité donne à entendre les témoignages marqués par l'empreinte de l'histoire et l'expérience vécue. L'expérience, quand elle se fait mémoire distanciée, est de nature autre puisqu'elle se construit dans le cadre social et institutionnel dans lequel elle se déroule. C'est de cette interpénétration de l'histoire et de la mémoire que les témoignages ont été produits dans l'après-coup et à travers des parcours croisés.

\section{Des parcours croisés}

Le plateau est constitué de trois témoins: une femme et deux hommes. Avant leur arrivée en Algérie et au Maroc, Paule Gauneau et Jacques Ladsous suivent à Montpellier une formation d'éducateur spécialisé et de travail social mise en place à l'Institut de Psycho-pédagogie médico-sociale par le Pr Lafon. André Heinrich, fait "ses classes" au sein du groupe Lyautey. Chacun d'entre eux a vu son expérience, dans ces années d'après-guerre, marquée et traversée par des engagements différents et communs. Le lien se crée autour du militantisme chrétien et de leur foi en Dieu. Cetre expérience de l'engagement collectif, à travers le militantisme chrérien, donne du sens à leur parcours professionnel et personnel dans la nécessité pour eux de faire quelque chose pour les délaissés, les déclassés, les marginaux. Ils forment, en ce sens, une communauté de jeunes individus unis par une même ambition : être sur le terrain pour répondre à une demande sociale et urgente d'encadrement de l'enfance et de la jeunesse en grande difficulté affective, sociale et économique. Une jeunesse à la limite de la délinquance, en tout cas identifiée comme telle. Le fait que ces jeunes gens soient issus des mouvements de jeunesse chrétienne a son importance. Elle dévoile des parcours très liés aux mouvements d'éducation populaire, au scoutisme, qui ont forgé une représentation du monde et des hommes - entraide,

5. Michel Foucault, Le souci de soi, t. 3 : Histoire de la sexualite, Paris, Gallimard, 1984. 
solidarité, coéducation, rénovations pédagogiques, justice sociale - en interaction avec leur pratique professionnelle.

Quand ils débarquent à Alger et au Maroc, c'est en professionnels de l'aide à l'enfance avec le désir de participer à une aventure professionnelle nouvelle mise en place d'un projet institutionnel pensé à partir de la métropole - sur un territoire inconnu face à des populations de cultures diverses avec des représentations qui s'inscrivent dans le temps du colonialisme, c'est-à-dire : cet ailleurs c'est la France.

Enfin, pas tout à fait ! Certes l'Algérie est un territoire français constitué de trois départements et est, en théorie, un prolongement outre-mer, de la République française. La III République va choisir, en Algérie, de mêler deux dimensions : intégration des terres, ségrégation des hommes. L'idée d'une "plus grande France " au nom des valeurs universalistes, en métropole, va renforcer le régime républicain et par conséquent le pouvoir de l'État, en accentuant l'imaginaire national, dans un destin universel afin de promouvoir le "modèle français ", héritier de la révolution.

Le système social français en Algérie est divisé en deux catégories distinctes : d'un côté les Musulmans et de l'autre les Européens. Les rapports s'inscrivent alors dans une dimension de domination principalement fondée sur la croyance au dogme de l'homme universel par les européens. Cette position n'est que théorique et se construit dans la pratique par la mise en place de politiques et de comportements ségrégationnistes assis sur un arsenal de dispositifs législatifs et réglementaires : décret Crémieux, code de l'indigénat, naturalisation automatique, institution d'une instruction séparée avec la création d'une école spécifique aux indigènes. ${ }^{6}$ La question de la scolarisation des enfants musulmans ne répond pas au projet initial de la $\mathrm{III}^{e}$ République dans la mise en œuvre des politiques éducatives. L'instauration d'une école publique gratuite, laïque et obligatoire est bien entendu mise en place en Algérie, à la différence fondamen-

6. Le décret Crémieux du 24 actobre 1870 stipule que les israélites indigènes des départements de l'Algérie sont déclarés citoyens français, leurs statuts réel et personnel seront réglés par la loi française. L'adoption du décret sur l'indigénat du 7 octobre 1881 est à mettre en relation avec ses nouvelles mesures Les Juifs doivent prouver leur indigénat, prouver qu'ils étaient nés en Algérie avant la conquête ou nés de parents y résidant à cette époque. Les juifs du Sud identifiés comme des Berbères ou des orientaux d'origine ne purent bénéficier du décret Crémieux et eurent le même statut que les indigènes musulmans soumis au code de l'indigénat. La loi du 26 juin 1889 impose aux Européens la naturalisation automatique pour tout étranger né en Algérie. Le sénatus consulte de 1865 attribuait aux indigènes (musulmans et juifs) la nationalité française. Suivant que l'on soit indigène ou étranger, le terme de naturalisation n'est pas soumis au même dispositif législatif. Le caractère individuel de la naturalisation en fait une prérogative gouvernementale discriminante en matière de nationalité et de citoyenneté. Le décret Crémieux, critiqué, permit d'écarter toutes les propositions suggérant l'attribution de la citoyenneté aux indigènes musulmans. 
tale qu'à sa marge est créée une école spécifique aux indigènes jusqu'en 1949. Elle diffuse une manière d'être exotique pour les enfants algériens : rapport au temps, à l'espace, au corps, à la propriété. L'idée que le savoir doit être dispensé dans des institutions spécialisées, par des individus formés à cette fin, que son mode de transmission doit être écrit et non oral et qu'il doit être le même pour tous, ces obligations qui sont étrangères à la culture paysanne comme à la culture théologique musulmane, ne présentent aucun intérêt et aucun caractère de nécessité du point de vue de leur logique de transmission du savoir. Il est aisé d'expliquer que ces savoirs sont du côté de l'urbanité et non de la ruralité, du côté de la modernité et non de l'immuabilité, et que ces antagonismes en apparence abstraits renvoient en réalité à des définitions discordantes du savoir qui elles mêmes renvoient à des conflits de groupes. Entre 1890 et 1940, l'histoire de l'école indigène enregistre de multiples réformes qui vont peu à peu l'orienter vers une identification à l'école européenne, plus particulièrement dans les villes. Sous la pression des colons les programmes spéciaux seront maintenus en milieu rural. La mise en place des écoles gourbis puis des centres ruraux d'éducation le terme d'expérience leur est souvent associé, ce qui montre leur caractère atypique - n'infléchit pas le cours de l'évolution scolaire en Algérie. Constat édifiant, l'impossibilité de mettre en place une des devises de l'école républicaine : obligatoire. À la veille de l'indépendance environ $90 \%$ des enfants européens en âge scolaire sont scolarisés contre $15 \%$ des enfants algériens ${ }^{7}$.

Ce contexte général de l'éducation des enfants en Algérie n'est pas sans lien avec les missions et l'institutionnalisation de la protection de l'enfance en Algérie. Dans ce secteur persiste un décalage entre la réalité en Afrique du Nord et la métropole. En Algérie, par exemple, la réglementation, et surtout son application en termes de rééducation et de protection des mineurs, subit un écart dans les dispositions prises en métropole. La charte de la jeunesse délinquante du 2 février $1945^{\circ}$ qui instaure la prise en charge des enfants de justice, dans le cadre de l'éducation, de la pédagogie, de la psychologie, n'entre en vigueur qu'en $1951^{\circ}$. Les éducateurs qui débarquent de métropole au début des années 50 se trouvent plongés dans un espace territorial, social et politique "clivé ". Ce clivage se cristallise de différentes manières. Tout d'abord, un fort accent est mis sur la spécificité de la déviance juvénile en interrelation avec le territoire immense et très hétérogène dans sa composition sociale. Ce qui serait plus juste,

7. A. MERAD, "Regards sur l'enseignement des musulmans en Algérie 1880-1960", Confluent, $\mathrm{n}^{\circ} 32$ et 33, juin-juillet, 1963, pp. 596-646.

8. Une loi précédente, celle de 1912 qui instituait des juridictions spéciales et le régime de liberté surveillée et substituait aux mesures répressives des mesures éducatives, fut appliquée en 1935.

9. Raymond SUlTAN, "L'Éducation surveillée en Algérie 1945 à 1962 ", Revue d'histoire de l'enfance irrégulière, $\mathrm{n}^{\circ} 1,1998$, La protection de l'enfance, pp 156-165. 
d'ailleurs, dans son opposition ruraux/urbains. Ce qui nous renvoie immanquablement à la question scolaire. L'autre élément très important c'est le rapport de force démographique entre les populations musulmanes, assujetties " invisibles " du point de vue juridique et politique, même si la loi de 1947 octroie la citoyenneté par le droit de vote aux algériens. Certainement trop tard puisque le 8 mai 1945 les nationalistes organisent un défilé pour célébrer la défaite de l'Allemagne nazie en scandant " $A$ bas le fascisme et le colonialisme" ou "Vive l'Algérie libre et indépendante ". Le processus vers l'indépendance est en route et la question de l'assimilation n'est plus vraiment à l'ordre du jour. Le paysage institutionnel du secteur de la protection de l'enfance est à l'état de balbutiement et son rattachement à la sécurité générale -administration pénitentiaire et du maintien de l'ordre- lui confere un visage très différent de la métropole. En effet, l'ordonnance de 1945 institue une direction autonome de l'éducation surveillée au sein du ministère de la justice chargée de mettre en œuvre les décisions éducatives prises par les juridictions compétentes à l'égard des mineurs. Il faudra attendre 1955 pour que l'éducation surveillée devienne un service autonome. Il faut ajouter à cela une présence sur le territoire de l'Algérie d'un secteur privé catholique ainsi qu'un réseau associatif mais à l'inverse de la métropole le secteur public de la protection de l'enfance se développe davantage. La situation de guerre dans laquelle le pays est engagé dès 1954 a des conséquences sur l'exercice et les missions du métier, ainsi que sur l'implication et les engagements des témoins.

Jacques Ladsous arrive en Algérie en 1950 après un passage rapide dans l'enseignement, expérience éphémère qui met le doigt sur sa résistance à l'autorité et la hiérarchie mais pose les jalons d'un questionnement : que faire pour les marginaux? Puis, il travaille en centre d'observation crée par le Pr Lafon à Montpellier qui l'amène à suivre la formation de l'IPPMS ${ }^{10}$ pendant un an. Les relations entre les deux hommes sont complexes et conflictuelles. C'est la vision de l'adolescent qui est au cœur de leur antagonisme. Pour Jacques Ladsous les adolescents doivent assister aux réunions de synthèse car l'observation doit être participative. Cette conception est en totale opposition avec sa direction. Il donne sa démission et rejoint l'Algérie où il prend en charge, à Chréa, une communauté d'enfants. Les communautés d'enfants ne lui sont pas inconnues. Quand, à 17 ans, il s'engage dans la Résistance - fortement imprégné de la figure ouvrière paternelle - il s'occupe dans le maquis d'enfants juifs cachés par leurs parents. C'est le début de son expérience éducative.

Sa période algérienne confirme sa résistance à toutes formes d'oppression et d'empêchement de liberté et l'amène à s'opposer à la politique coloniale menée par l'état français. La communauté qu'il dirige se trouve sur un territoire isolé et

10. Institut de Psycho-pédagogie médico-sociale. 
stratégique pour les opérations de "pacification " et de maintien de l'ordre colonial dans sa lutte contre l'armée de libération. Il refuse que l'armée française s'installe sur ce territoire. Il est arrêté et emprisonné.

L'Algérie montre une réalité qui le questionne tant du point de vue sociétal que professionnel. Comment se fait-il que cette communauté d'enfants n'accueille pas de jeunes musulmans et qu'une seule classe sociale soit vraiment représentée ?

"C'était comme s'il n'existait pas d'oedipe au niveau des familles musulmanes et j'ai commencé à m'interroger sur la maladie mentale comme phénomène d'une société riche ".

Il poursuit alors sa réflexion sur l'inadaptation dans ce contexte colonial comme réalité assez simple. Selon lui, les individus sont maintenus dans une vie marginale par un pouvoir blanc colonisateur et donner la parole aux individus participe du processus d'effacement de l'inadaptation.

Si Jacques Ladsous a une perception sociale et politique de la colonisation, ce n'est pas le cas de Paule Gauneau à son arrivée en Algérie. C'est la même année, 1950, qu'elle débarque à Alger après l'obtention du diplôme d'État d'infirmière et d'assistante sociale et après avoir suivi la formation d'éducatrice à Montpellier. Elle rejoint l'hôpital Mustapha où une chaire de neuropsychiatrie vient de se créer à l'initiative du Pr Lafon à Montpellier et du Pr Sutter à Alger. C'est la naissance du premier CMPP" dans le département d'Alger. Elle n'arrive pas seule. Dans le même temps, une équipe de Montpellier ouvre le premier centre de rééducation de garçons intelligents caractériels. C'est donc dans l'enthousiasme que tout ces jeunes gens foulent le sol algérien très convaincus professionnellement de la mission à accomplir dans un cadre nouveau à construire. Paule Gauneau se décrit comme " une petite française ignorante et naïve ne connaissant rien à la situation algérienne. Pour moi c'était un département français comme les autres. ". Elle explique que ses premières impressions sont bonnes mais assez décalées du local : "Nous arrivions avec un projet pédagogique comme si tous les Algériens étaient des petits français. ". L'essentiel, selon elle, est de faire son travail et d'être pour la population le lien accessible. Cette accessibilité prend sa source, sans doute, dans sa proximité à l'autre acquise dans l'engagement chrétien, à la JEC ${ }^{12}$. Elle se sent imprégnée de cette mouvance et souligne : " j'étais ressentie comme peut être une bonne sœur, le pauvre c'était un être concret ». A partir de 1954, les moments difficiles commencent avec les attentats, la répression. La jeune femme est protégée dans un environnement professionnel et amical européen. L'histoire la rattrape en mai 1956. C'est la

11. Centre médico-psycho-pédagogique.

12. Jeunesse étudiante chrétienne. 
nuit, Alger est bouclée à cause du couvre feu. Elle est emmenée, avec ses collègues, au commissariat central d'Alger. Elles sont ensuite séparées en groupes de huit et dispersées dans différents commissariats de la capitale. Elle apprend à ce moment l'injonction qui leur est faite : un groupe de militaires et de policiers doivent perquisitionner dans la Casbah et les assistantes sociales sont chargées de fouiller les femmes. Toutes sont fonctionnaires de l'État et censées obéir aux ordres. Elle refuse, comme d'autres, et passe la nuit au commissariat avec le risque de révocation. Elle est considérée comme anti-française et pro-fellagha sans subir de représailles. Elle est toutefois mal jugée et mise à l'écart par certains collègues. L'affaire est restée secrète dans le respect des consignes du Directeur de la santé. "On ne pouvait pas en parler, je ne sais plus si j'en ai parlé autour de moi ".

André Heinrich arrive au Maroc en 1949 dans la banlieue de Casablanca, au centre d'accueil des jeunes musulmans, comme éducateur puis adjoint du chef de centre. Les enfants accueillis, suite à une grande sécheresse qui amplifie la misère locale, sont dans un état sanitaire déplorable. Ce sont des enfants des rues, des petits délinquants, âgés de 7 à 16 ans pris en charge en internat, institution scolaire et ateliers sportifs dépendant du service de la jeunesse et des sports. Selon André Heinrich, «la grande BA du service». A cette époque les milieux du scoutisme font des interventions au centre (section de l'enfance délaissée). En 1952 le centre d'accueil devient centre d'accueil et d'observation suite au détachement de Jacques Selosse du ministère de la justice française. Le service devient Bureau de l'enfance délaissée et de l'éducation surveillée. Le Maroc et l'Algérie ne connaissent pas les mêmes affres de l'histoire et le processus d'indépendance au Maroc n'a pas les mêmes conséquences sur les individus. Ceci étant dit, la réalité coloniale est tout aussi prégnante au Maroc. La catégorisation des populations n'est pas un leurre et des écarts énormes existent entre les enfants des villes et des campagnes, entre les enfants musulmans et européens. Cette situation interroge sa foi catholique en même temps que la découverte de l'islam au contact des musulmans. Il reste au Maroc jusqu'en 1969 après avoir occupé différentes fonctions et missions.

Son histoire en terre marocaine ne s'achève pas pour autant. En 1981, il est sollicité pour une semaine de séminaire sur la thématique des interventions en milieu ouvert au centre national de formation des éducateurs de Yakoub el Mansour. En 1982 il participe à l'élaboration de programmes de formation. Dès lors, les allers et retours entre les deux pays n'ont pas manqué. En particulier entre 1992 et 1996 où il assure une semaine par an la formation des directeurs de différents établissements et services de sauvegarde de l'enfance du Ministère de la Jeunesse et des Sports, dans le cadre de la formation permanente. 


\section{Dans l'entrelacs de l'engagement et des missions institutionnelles}

Il n'est pas question ici de faire la genèse de la profession. Toutefois, il est essentiel de rappeler que la période de la guerre et de l'après-guerre institue la professionnalisation des éducateurs issus du scoutisme et des camps de jeunesse, et favorise la création d'écoles et de formations à l'initiative de religieux (l'Abbé Plaquevent à Toulouse) puis sous l'impulsion de la neuropsychiatrie ( $\operatorname{Pr}$ Lafon Montpellier). L'éducateur se spécialise très vite, aidé en cela par des associations professionnelles (ANEJI) ${ }^{13}$. Ses missions sont diverses : éducative et pédagogique à travers l'animation d'ateliers pour jeunes délinquants avec pour objectif de les remettre sur le chemin du travail, la prise en charge de jeunes déficients intellectuels, de jeunes atteints de troubles du comportement placés par leur famille en institution... Bref, la mission institutionnelle majeure de l'éducateur se forge dans la relation à l'autre et la mise en ouvre de dispositifs pour remédier à l'inadaptation des jeunes. Pour Jacques Ladsous et André Heinrich c'est une quête sans répit. Tout d'abord en se questionnant sur le sens même de l'inadaptation des individus. Qui produit celle-ci ? Pour Jacques Ladsous la réponse est claire : c'est le système de relégation sociale et coloniale qui en est à l'origine. A cette époque, la pédagogie de Makarenko, basée sur la coopération, influence la pratique de Jacques Ladsous. Pour lui, une coopération participative des jeunes est fondamentale parce qu'elle porte leur parole, ce qui l'amène à poser un regard analytique sur les jeunes : "dans une perspective de " conscientisation ", offrait un outil extraordinairement intéressant [...] les jeunes n'étaient pas des objets mais des sujets et un sujet ne peut être sujet que s'il est conscient ". Mais c'est aussi une vision politique qui s'impose à lui. Le centre dont il a la charge devient un lieu de rencontre des opposants au colonialisme et un laboratoire expérimental où les théories et les pratiques développées ont permis d'apporter des réponses aux jeunes en souffrance sociale. Pour ceux dont les troubles psychiques ont été plus complexes une collaboration s'est engagée avec le laboratoire de psychologie de la faculté d'Alger. Jacques Ladsous reçoit tous les cas qui ne trouvent de place ailleurs, il qualifie son centre de "cour des miracles " alors que l'époque catégorise l'inadaptation. Il met en cause la formation des éducateurs comme formation " inadaptée ", voire dangereuse :

"les formarions tendent à compartimenter [...] alors qu'en prenant le tout venant et que l'on crée une certaine communauté, on arrive à des résultats où le phénomène d'inadaptation n'est plus reconnu comme tel ".

Dans ce contexte précis de fin de colonisation l'état français poursuit la mise en ouvre de modèles pensés à partir de la métropole dans la continuité des

13. Association nationale des éducateurs de jeunes inadaptés. 
politiques et des dispositifs législatifs de catégorisation des populations. L'expérience algérienne de Jacques Ladsous participe d'une double construction, dans les choix qu'il entreprend, du point de vue individuel et collectif. L'immersion dans un contexte colonial lui permet de théoriser et d'appliquer de nouvelles formes éducatives impensées et impensables vues de la France et produit un déplacement de ses missions à la marge des prescriptions de son institution. Ce qui peut être considéré comme une remise en cause de la mission institutionnelle s'avère dans certaines circonstances l'application même de cette mission. En effet, quand Paule Gauneau décide de ne pas fouiller les femmes de la Casbah, c'est en totale adéquation avec les attributions professionnelles qui sont les siennes, c'est-à-dire la prise en charge des personnes en souffrance sociale ou psychique. L'état lui assigne une autre mission, celle de sécurité nationale, tâche attribuée aux forces de police et de maintien de l'ordre. Son refus de s'exécuter marque un acte d'opposition pour celui qui le prescrit mais pour l'assistante sociale " la fouille " ne relève pas de ses missions professionnelles. Au-delà d'une position humaniste et morale, voire politique, c'est en réalité sa profession et sa mission de service social en Algérie qu'elle assume et protège.

André Heinrich va voir sa mission s'élargir avec la promulgation de textes législatifs et réglementaires relatifs à l'accueil des jeunes délinquants qui leur ouvrent les portes du centre. La période de 1953 à 1956 accentue la coopération avec la justice, la police et les services sociaux et voit la création de sept nouveaux centres (observation, éducation surveillée, semi-liberté, liberté surveillée). Parallèlement, la formation du personnel s'engage et les premiers documents bilingues sont diffusés. Après l'indépendance, en 1958, Abdelhatif el Backa est nommé chef du bureau qui devient Bureau de l'action éducative et de l'enfance inadaptée. Dans le même temps, André Heinrich change de fonction et prend la direction du centre d'observation de Tanger (liberté surveillée). Durant ces années le manque d'éducateurs musulmans formés se fait fortement sentir. C'est ce manque qu'il va tenter de combler comme un passeur de savoirs pour assurer la relève. Dans le centre un seul moniteur marocain travaille avec l'équipe française. Selon lui, c'est une volonté politique de ne pas permettre l'intégration des marocains à la formation et au métier ainsi qu'une méfiance indéniable des jeunes français face aux personnels marocains. Le problème découle, également, des populations qui fréquentent les centres (très peu mixtes). "On ne mélange pas les serviettes et les torchons ". Après 1956, il constate assez peu de ruptures. Le poids du modèle français est très présent et peu de bouleversements sont opérés. Cependant, un processus de formation s'engage, processus auquel il participe activement. Il suit une année de formation à Vaucresson, dispositif mis en place par le secteur associatif en France. $A$ son retour son souhait est " de former des camarades marocains qui prendront la relève" avec l'acquisition théorique, pratique et humaniste nécessaire à l'exercice du métier. En 1962, il est chargé de cours au centre de formation des éducateurs à l'invitation du mi- 
nistère d'Abelhatif el Backa avec pour mission de poursuivre le travail engagé en l'adaptant, au service central à Rabat et à l'école de formation de Yakoub el Mansour. En 1965, à Casablanca, les émeutes lycéennes du 23 mars réprimées dans le sang marquent une période de turbulences dans la société marocaine et dans les missions éducatives des professionnels du secteur. De nombreuses questions émergent sur le bien fondé du rattachement au service de la promotion populaire et promotion masculine. Cependant, cette même période voit s'opérer un développement important des dispositifs et moyens mis en œuvre pour la formation professionnelle des jeunes en collaboration avec le ministère du travail et les coopératives ouvrières de production, par exemple. La coopération avec la France se maintient avec l'accueil de fonctionnaires français du ministère de la Justice pour les sessions de formation. De nombreux éducateurs et directeurs marocains partent en France ou en Belgique pour des colloques et des cycles de formations. Parallèlement, l'ANEJI se développe au Maroc. En 1969, l'expérience marocaine se termine et André Heinrich rentre en France au CREAI $^{14}$ du Sud-est. Sa mission principale de " passeur " est assurée.

\section{Le retour : d'une rive à l'autre}

La question des "rapatriés ", et par rebond celle du " retour", est encore une question vive dans les mémoires individuelles et familiales, plus particulièrement des européens et juifs d'Algérie ${ }^{15}$. Si le terme de "rapatriement" est largement contesté, le lieu commun de "retour des pieds-noirs " fait passer à l'arrière-plan les réalités algériennes de l'époque. En effet, les éléments fondateurs de l'identité pied-noir sont récents et parfois falsificateurs. La population française d'Algérie est la résultante de populations "mélangées " qui se sont parfois opposées les unes aux autres et, dans tous les cas, hormis les français de souche, sont composés d'immigrés. L'exil a complètement renouvelé et modifié l'espace social qui régissait leur vie en Algérie. Le rapatriement n'est pas qu'une simple migration. Il cristallise un paradoxe fort, celui d'une appartenance à la fois fictive et réelle à la France ${ }^{16}$. Le déracinement, l'exil, ont provoqué des blessures morales et affectives qu'exprime le culte du souvenir, c'est à dire la nostalgie. Ils ont construit une communauté au sens strict d'un " entre soi » sur le sol de la France. Une communauté métaphorique d'un territoire unifié et symbolique qui s'est bâti dans un espace hors sol. Le retour n'est qu'une digression discursive et politique d'une pseudo-réalité qui n'a jamais existé.

14. Centre régional pour l'enfance et l'adolescence inadaptée.

15. Michèle BaUSSAnT, Pieds-noirs : mémoire d'exils, Paris, Stock, 2002.

16. Ibid. 
Il est évident que pour les français de métropole qui arrivent en Algérie et au Maroc au début des années 1950, pour des raisons essentiellement professionnelles, le retour s'est entrepris dans une autre réalité. D'abord, il s'est agi d'un vrai retour sur une terre connue, la leur. Leur position de jeunes français venus de la métropole ne peut, en aucun cas, les identifier aux pieds-noirs. Cependant, les années passées en Algérie et au Maroc ont produit des effets lors de ce rapatriement, pour reprendre l'expression d'André Heinrich. Il s'inscrit pour Jacques Ladsous et Paule Gauneau au cœur du contexte de guerre dans lequel l'Algérie est plongée, et ce pour des motifs différents. L'engagement militant contre le pouvoir colonial et la torture ont mené à la prison Jacques Ladsous et à un retour forcé en métropole. Ce qui a pour conséquence de le placer dans une situation délicate auprès de ses pairs dont certains lui reprochent son manque de loyauté envers l'état français. Après quelques mois difficiles, il rencontre Jean Pinaud (inspecteur de la santé publique et de la population) et le seconde dans le pilotage de la première école d'éducateurs à Épinay-sur-Seine. Suite à des désaccords pédagogiques profonds, il rejoint trois ans plus tard les CEMEA ${ }^{17}$ comme permanent de l'association. Que reste t-il de son expérience algérienne ? Certainement une impossible séparation entre l'homme privé et le professionnel ainsi qu'une empreinte indélébile à la cause des dominés de tous ordres.

Paule Gauneau rentre en France en 1957 après une mission de trois ans comme fonctionnaire du gouvernement général d'Alger. C'est-à-dire, dans le contexte de l'époque, au service d'un département français comme un autre. Pas tout à fait. Elle découvre à son retour qu'aucune prise en compte des états de service en Algérie n'est possible. Ce constat et son expérience en Algérie l'interrogent sur ce territoire qu'elle considérait, à son arrivée, comme français à part entière et qui se révèle être une expansion coloniale avec ses limites et séparations dans les statuts accordés aux individus, citoyens ou sujets français.

Ce passage, plus ou moins long, par l'Algérie et le Maroc, a laissé des traces dans les vies des témoins. C'est là-bas que la figure de l'altérité a pris forme. L'expérience de la différence, des balbutiements d'une profession émergente, des projets à structurer, à inventer, à explorer, participent d'une réflexion plus large sur la pédagogie et l'aide à l'enfance ainsi que sur la nécessité de construire des dispositifs de formation initiale et continue et d'analyse de pratiques des professionnels.

Les témoignages éclairent un contexte singulier, celui de territoires colonisés où se sont montés des projets socio-éducatifs nouveaux. Pour chacun des témoins ces années passées en Algérie et au Maroc sont porteurs de multiples significations et implications. Elles sont à la croisée des missions à mettre en place, à inventer au fil du temps et des espaces. Elles sont à la croisée des ques-

17. Centres d'entraînement aux méthodes d'éducation active. 
tionnements individuels et collectifs sur une vision du monde et des Hommes. Elles sont à la croisée des engagements et des combats à mener pour une profession qui se construit, $s$ 'institue mais aussi pour une idée de la justice sociale et de la liberté. Le fil conducteur entre ces trois parcours professionnels et humains puise avant tout sa source dans le combat. Un combat qui s'inscrit dans l'institutionnalisation d'une profession et dans la sauvegarde de valeurs inspirées d'une forme d'humanisme chrétien.

Patricia BESSAOUD-ALONSO 